\title{
STABILITY AND FLUCTUATIONS IN A THREE SPECIES SYSTEM: A PLANT WITH TWO VERY DIFFERENT POLLINATORS
}

\author{
F.R. MOMO* - J.E.URE \\ Universidad Nacional de General Sarmiento \\ J.M.Gutiérrez 1150 (1613) Los Polvorines, Argentina \\ (phone: +54-11-4469-7543; fax: +54-11-4469-7500) \\ *Corresponding author \\ e-mail:fmomo@ungs.edu.ar \\ (Received $20^{\text {th }}$ October 2008 ; accepted $24^{\text {th }}$ November 2009)
}

\begin{abstract}
We present a multi-species model of one plant with two nectivore pollinators. This system has mutualism between the plant and the pollinators, and competition for food between pollinators. Both pollinators are different: the large pollinator can consume all the nectar offered by the plant in each visit; it permits the genetic interchange between plants from different patches, so, it avoids self-fertilization problems. The small pollinator consumes only a fraction of the flower nectar but the seeds produced by its pollination are less successful. The model gives different dynamics depending on both the differences between the pollinators' growth rates and the inbreeding effect. Stronger oscillations occur when the growth rates are very different. Paradoxically, a high inbreeding causes an increment in populations. The three species system is biologically stable and the asymmetry between pollinator species results in different roles that enhance the global stability and persistence of the system.
\end{abstract}

Keywords: Pollination; mutualism; population dynamics; competition; mathematical models

\section{Introduction}

Ecological relationships usually involve several interacting species in a complex network. Notwithstanding this, most studies have focused on two species interactions. Predation, competition, parasitism, and mutualism are examples of systems extensively studied and modeled by ecologists as two species interactions. However, none of these relationships is in actual fact restricted to two species; competition for instance is clearly a three-element system because it involves at least two competitors and one resource that typically is another species, an ignored fact in the traditional LotkaVolterra approximation [27].

Mutualism, in turn, is considered as a relationship in which both participants experience some net benefit. Mutualistic interactions occur along the entire range of biological organization and on all timescales $[4,17]$. These two relations, mutualism and competition, are apparently opposed to each other, although they may be seen as extremes of an interaction gradient with different benefit:cost ratios [4]. In fact, many interactions are a mixture of different aspects that involve competitive, antagonistic and mutualistic interactions [38]. From the benefit:cost perspective, it becomes clear that the outcomes of mutualism and competition must be extremely variable, and not simply plus/plus or plus/minus interactions; in fact, outcomes of inter-specific relationships vary with the abiotic and biotic setting in which the interactions take place, and there are several factors that influence mutualism [16]. 
A common example of mutualism is pollination [3]. The plant-pollinator relationship happens between free-living species and, as in several mutualistic associations, one partner performs some action (a service) that benefits its associate receiving some payoff for doing so. These rewards may be some kind of food, for instance nectar [10, 21], pollen [11], or even seeds or ovules that the pollinator larvae eat $[1,9,15]$. Providing rewards has a cost for the plant. For example, up to $37 \%$ of the photosynthate that the milkweed, Asclepias syriaca, assimilates during flowering is used to produce nectar [33]. Nectar can contain as much as $5 \mathrm{~kJ} \mathrm{ml}^{-1}$ of energy and provide half of the calories that a hummingbird needs [26].

The evolutionary significance of pollination has long been recognized [7, 23, 26, 29, 31]. These authors pointed out its role in the evolution of species by natural selection and, specially, its effect on morphological adaptations. A wide range of evidence suggests that some flower traits are specific adaptations that facilitate or enhance the pollinator visits $[8,41]$. Pollinators have a great influence in gene flux between plant populations, affecting their genetic structure [8, 40,41] and changing the seed production and the number of established seedlings [20,31].

Despite the obvious two-side benefit in the plant-pollinator relationship, there is more in this system than one can see at a first glance.

The plant-pollinator relationship is rarely limited to a couple of species. It is more frequent to find processes, which involve several pollinators that compete between them for obtaining nectar, pollen or oviposition sites [5, 6, 13, 20] describe 23 species of pollinator insects for the Kiwi (Actinidia deliciosa) flowers, including 11 species of Diptera, five of Hymenoptera, and four of Coleoptera. [19] describes 11 species of bees, 12 species of butterflies and six species of flies that work as pollinators for the Mediterranean shrub Lavandula latifolia. According to [11], Hormathophylla spinosa (Cruciferae) has at least 70 visiting species of insects, grouped in two guilds: nectarivores (mainly small flies and ants) and pollinivores (bees and hover flies). Although insects are the main pollinator animals, many other animals may do so as well. For example humming-birds, bats, small rodents and marsupials are all well known pollinators (see for example $[11,25]$ ).

It is then clear that during the process of pollination, several pollinators may interact among themselves. It is highly probable that different nectarivores or pollinivores compete for food, introducing a new element in this ecological web. The central questions then are, why don't single plant species have a single pollinator, for instance the most efficient, or the "cheapest" in terms of energy costs? How can a multispecies system be stable in ecological and evolutionary terms? Or, does a "best" pollinator exist?

In this paper we use a mathematical model to study the population dynamics of two pollinators and their resource, and the viability of this system in an attempt to provide answers to the above questions.

\section{The mathematical model}

We considered the simplest multi-species model: one plant and two pollinators. However, both pollinators differ in terms of home range, pollination efficiency and energy cost for the plant. In this way, the system represents mutualistic interactions (pollinator and plant) and competition (pollinators). The mutualism has different characteristics depending on the pollinator but competition is limited to resource 
exploitation because we assume no interference (never two pollinators visit the same flower simultaneously)

The model has the following assumptions:

a) There is only one species of plant, which bears one flower per individual per year. This restriction is unrealistic but necessary to avoid introducing effects of clumping or repeated visits to the same plant.

b) This flower produces a certain amount of nectar, which is renewed daily.

c) The plant is spatially distributed in patches.

d) There is a large pollinator (e.g.: a hummingbird), which has a wide movement range (can fly between patches), and consumes the total amount of nectar in each flower visit.

e) There is a small pollinator (e.g.: an insect), which has a limited movement range, and cannot fly between patches. This small pollinator consumes only a fraction $1 / f$ of the nectar of each flower it visits.

f) The flowers pollinated by the large pollinator produce in the next generation stronger and more numerous plants, due to a more intense genetic exchange between flowers from different patches.

g) Pollination is limited to either pollinator. Only the pollinators pollinate the plants, pollination by wind or any other means is neglected.

h) The plants reproduce annually and have discrete generations.

i) Pollinators reproduce annually but have overlapping generations.

Let $A$ be the density of the small pollinator's population, $P$ the density of the large pollinator's population, $N_{t}$ the total number of flowers, at generation $t$. We have the following system of discrete equations representing the competition between pollinators:

$$
\begin{aligned}
& P_{t+1}=P_{t} \times\left[1+r \times\left(1-\frac{A_{t}}{f \times N_{t}}-\frac{P_{t}}{N_{t}}\right)\right] \\
& A_{t+1}=A_{t} \times\left[1+s \times\left(1-\frac{A_{t}}{f \times N_{t}}-\frac{P_{t}}{N_{t}}\right)\right]
\end{aligned}
$$

Where $r$ and $s$ are the intrinsic grow rates for the large and small pollinators respectively. We assume that $r$, is smaller than $s$, given the well known relationship between growth rate and body size [32].

Regarding the flowers, we make a distinction between those pollinated by either the small or the large pollinator. This is due to the fact that the plants would not persist if only visited by the small pollinator, because of genetic inbreeding depression. So, if we represent the number of flowers pollinated by the small and the large pollinator respectively, $n_{A}$ and $n_{P}$, we have the following equations

$$
\begin{aligned}
& n_{A, t+1}=e_{A} \times\left(\alpha \times n_{A, t}+n_{P, t}\right) \times c_{A} \times c_{s a t}, \\
& n_{P, t+1}=e_{P} \times\left(n_{A, t}+n_{P, t}\right) \times c_{P} \times c_{s a t},
\end{aligned}
$$

Were $e_{A}$ and $e_{P}$ are the germination ability and survival rate of seedlings. These parameters are the product of four multiplying factors: flower fecundity, seeds survival, seeds germination ability, and seedling survival. Implicitly, this term introduces the assumption of a non-homogenous environment, because a plant being pollinated by the large pollinator has an advantage when exposed to a fluctuating or heterogeneous environment. Coefficients $c_{A}$ and $c_{P}$ are the ratio of $A$ and $P$ to the total $A+P$. 
The term $c_{\text {sat }}$ is a saturation function given by:

$$
c_{\text {sat }}=1-\exp \left(-d \frac{A+P}{N}\right)
$$

Where we have used $N_{t}$, the total number of plants

$$
N_{t}=n_{A, t}+n_{P, t}
$$

The function $c_{s a t}$ acts in such a way that the number of flowers in each generation does not depend on the pollinator density except when that density is low compared to the flower abundance.

The parameter $\alpha$ ranges between 0 and 1, indicating the negative effect of inbreeding on plants; smaller a represent stronger negative effects.

We ran the model for five thousand generations starting from an initial situation where the population of the small pollinator is an order of magnitude larger than the one for the large pollinator. This assumption is coherent with the biological characteristics of the pollinators and the accepted relationship between body size and population abundance. Initially, the number of plants pollinated by each pollinator was fixed and equal (two thousand).

We used several different values for a and for the $s / r$ relation; all the other parameters were kept constant: $e_{A}=1.2, e_{P}=1.4, d=3.0$

\section{Results}

Both pollinators have an equilibrium point that satisfies $A * / f+P^{*}=N$.

For plants, the equilibrium depends on several system parameters, in fact:

$$
\begin{aligned}
& n_{A}^{*}=e_{A}\left(\alpha n_{A}^{*}+n_{P}^{*}\right) c_{A} c_{s a t} \\
& n_{P}^{*}=e_{P}\left(n_{A}^{*}+n_{P}^{*}\right) c_{P} c_{\text {sat }}
\end{aligned}
$$

Stars in Eq. 7 and 8 symbolize fixed points (equilibria).

If we neglect $c_{s a t}$ variations, this is a homogeneous linear system that has a nontrivial solution only when its determinant is zero. In that case $n_{A} *$ is proportional to $n_{P} *$, their values depend on $A^{*}$ and $P^{*}$, and the system reaches a fixed equilibrium point.

Simulations show that there is no case where the actual model can present chaotic behavior, but it can converge with a monotonous or oscillatory behavior depending on the relationships among $\alpha, r$ and $s$.

When the $s / r$ relation is 2 (case of light asymmetry between pollinators), the system converges rapidly for high $\alpha$ (Figs. 1 and 2) as well as for low $\alpha$ (Figs. 3 and 4), and populations remain constant after 50 generations. When $\alpha$ has the smaller value, populations show a slight oscillatory behavior (Fig. 3). This is not surprising because the use of difference equations introduces an implicit delay [14]. Despite this transient dynamics, a soft asymmetry between pollinators ensures stability and a fast convergence. 


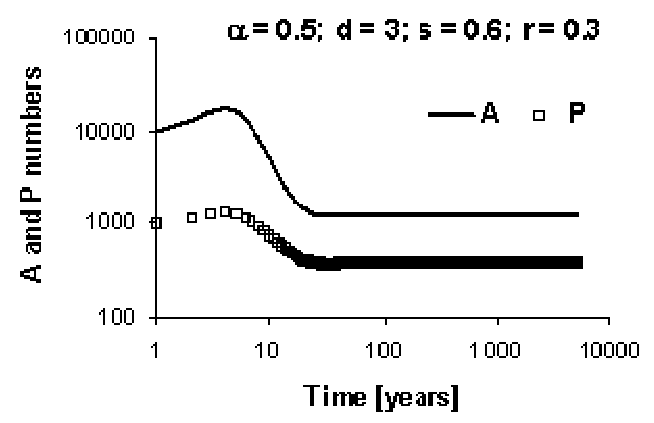

Figure 1. Dynamics of the two pollinator populations under conditions of slight inbreeding and low asymmetry: $\alpha=0.5$ and $s / r=2$. After 50 years, the system remains at stable equilibrium.

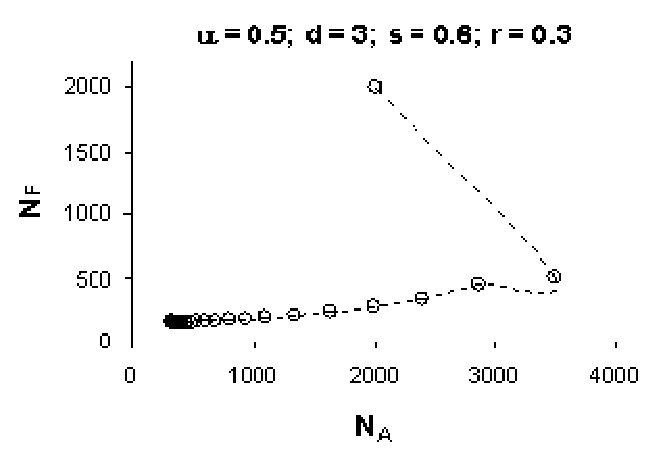

Figure 2. Phase space of plants pollinated by each pollinator for the conditions of slight inbreeding and low asymmetry. The starting point is [2000, 2000] and there is an attractor at $[331,163]$.

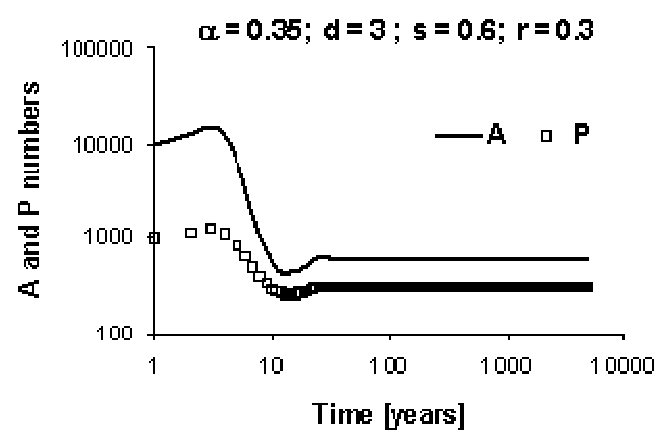

Figure 3. Dynamics of the two pollinator populations under conditions of slight inbreeding and high asymmetry: $\alpha=0.5$ and $s / r=8$. The system shows damped oscillations before reaching the stable equilibrium. 


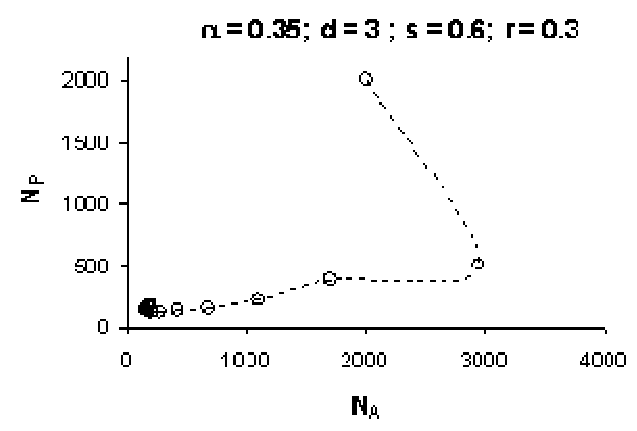

Figure 4. Phase space of plants pollinated by each pollinator for the conditions of slight inbreeding and high asymmetry. The starting point is [2000, 2000] and there is an attractor at $[729,486]$ that is reached after 100 generations of damped oscillations.

When the ratio $s / r=8$, that is, when the growing rate of the small pollinator is practically one order of magnitude greater than that for the large pollinator (a strong asymmetry), oscillations became more pronounced. For a large $\alpha$ the system stills converges towards equilibrium; exponentially after 100 generations or less (Figs. 5 and 6). But for small $\alpha$ (strong effect of inbreeding) the system converges very slowly towards an exponential growth of populations, through oscillations (Figs. 7 and 8). In this latter case, we can see that, surprisingly, a high inbreeding causes a population increment. If this behavior is transient, it is long lasting (i.e.: "supertransient"); at less 5000 years.

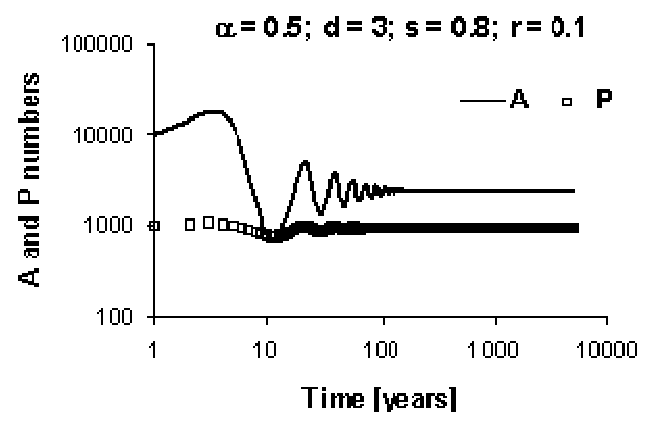

Figure 5. Dynamics of the two pollinator populations under conditions of slight inbreeding and high asymmetry: $\alpha=0.5$ and $s / r=8$. The system shows damped oscillations before reaching the stable equilibrium. 


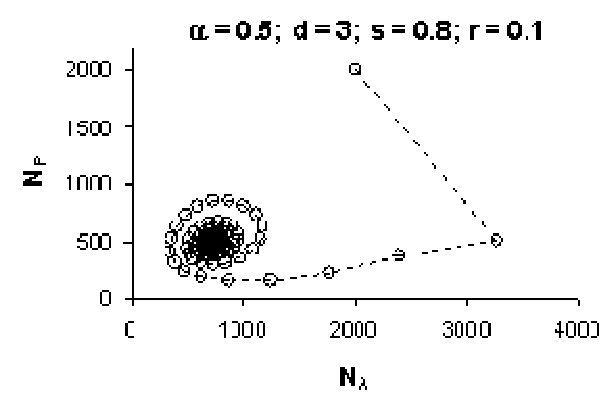

Figure 6. Phase space of plants pollinated by each pollinator for the conditions of slight inbreeding and high asymmetry. The starting point is [2000, 2000] and there is an attractor at $[729,486]$ which is reached after 100 generations of damped oscillations.

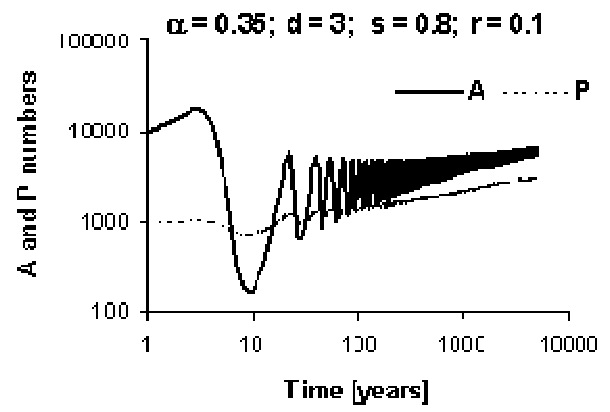

Figure 7. Dynamics of the two pollinator populations under conditions of strong inbreeding and high asymmetry: $\alpha=0.35$ and $s / r=8$. The system shows a long transient oscillatory behavior that converge to an exponential grow of both populations.

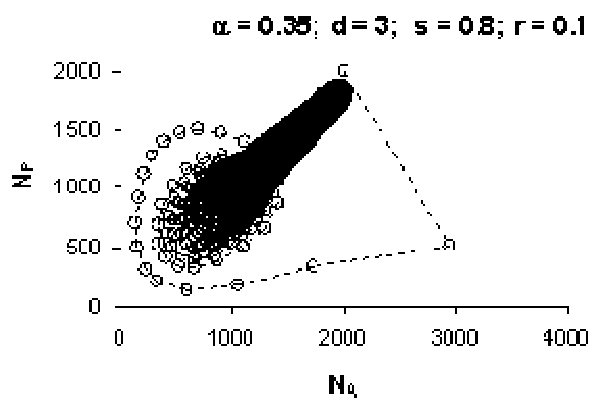

Figure 8. Phase space of plants pollinated by each pollinator for the conditions of strong inbreeding and high asymmetry. The starting point is [2000, 2000] and there is a long series of slightly damped oscillations. The tendency is to an exponential growth for both subpopulations. 
These different behaviors are summarized in Table 1 .

Table 1. Model behavior under different parameters sets.

Low $\mathrm{s} / \mathrm{r}$ ratio (2.7) High $\mathrm{s} / \mathrm{r}$ ratio $(8.0)$

High $\boldsymbol{\alpha}(\mathbf{0 . 5}) \quad$ Asymptotically stable Damped oscillations

Low $\boldsymbol{\alpha}(\mathbf{0 . 3 5}) \quad$ Stable with damped oscillations $\quad$ Growing with damped oscillations

\section{Discussion}

Traditionally, modeling of mutualism has been reduced to the case of only two interacting species [24, 28], or has been based on individuals [35] due to the importance of the individual pollinator behavior. Some mathematical approaches to mutualism have been recently published $[12,22]$ but with different objectives than ours.

Our simple model shows a rich spectrum of dynamics. The plant-pollinator system is unstable with the small pollinators only, except for particular situations in which the inbreeding effect is canceled $\left(\alpha * e_{A} \geq 1\right)$. On the other hand, large pollinators may coexist with the plant without the small pollinator but, due to its low growth rate, both populations would increase their numbers very slowly. It is clear in this context, that the introduction of a small pollinator benefits the large one because of causes an increase in the local plant populations. In this sense, our work shows the counterintuitive results in which pollinators may not compete but benefit form an indirect mutualistic relationship.

Our work shows also that realistic models of mutualisms need to involve the dynamics of three or more species. Attempts to do this suggest that the presence of a third species (predator or competitor) may stabilize some mutualistic interactions [3]. The persistence of assemblages of several pollinator species is important in the context of the conservation of biodiversity [36, 37]. It has been shown that there is a relation between pollination and the plant reproductive success, as well as a relation between pollinator's visits and the flowers' nectar offer [33, 34]. Most plants have life history traits that facilitate cross-pollination and ensure that beneficial genes can be transmitted relatively rapidly to successive generations [2]. Cross-pollination introduces genetic diversity into the population at a rate that enables the species to cope with a changing environment. This relationship between the pollination and the reproductive success of the plants has been broadly documented; for instance [30] have shown that sunflowers pollinated by bees, produce three times more seeds and four times more fruit by inflorescence than not pollinated ones. [13] found a similar effect in kiwi, where beespollinated plants bear fruits with weights $60 \%$ greater than not pollinated ones. [18] have shown that seed production was more broadly variable among wind-pollinated taxa than among animal-pollinated ones.

We cannot state that a "better" pollinator exists; the asymmetry between species results in different roles that enhance the global stability of the system. The least efficient pollinator brings a greater growth rate, while the pollinator with the slower rate 
of increase contributes to a better genetic exchange between plants and consequently, better chances of survival in non-homogeneous habitats.

The system shows a long range of stability for a wide variety of situations. Too intense inbreeding leads to fluctuations. The fluctuations are also induced by a strong difference in the growth rates of the two pollinators. When intense inbreeding coincides with the strong differences in growth rates, the system not only oscillates but also has growing populations. Therefore, a large population of a not efficient pollinator can benefit from a small population of a second kind of pollinators that gives the plant population higher genetic stability. That is, the presence of the second pollinator promotes the coexistence and beneficiates the first pollinator despite the competition between both.

Acknowledgements. We thank to Dr. C. Coviella for his comments and discussion, and to Dr. F. Carusella for her assistance.

\section{REFERENCES}

[1] Addicott, J., Bao, T. (1999): Limiting the coasts of mutualism: Multiple modes of interaction between yuccas and yucca moths. - Proceedings of the Royal Society of London Series B Biological Sciences 266: 197-202.

[2] Aizen, M., Ezcurra, C. (1998): High incidence of plant-animal mutualisms in the woody flora of the temperate forest of southern South America: Biogeographical origin and present ecological significance. - Ecologia Austral 8: 217-236.

[3] Begon, M., Harper, J.L., Townsend, C.R. (1996): Ecology: individuals, populations and communities. Third edition. - Blackwell, Oxford.

[4] Bronstein, J.L. (1994): Our current understand. of mutualism. - Q. Rev. Biol. 69: 31-51.

[5] Brown, J., Kodric-Brown, A. (1979): Convergence, competition and mimicry in a temperate community of hummingbird-pollinated flowers. - Ecology 60: 1022-1035.

[6] Brown, J., Kodric-Brown, A., Whitman, T., Bond, H. (1981): Competition between hummingbirds and insects in the pollination of two species of shrubs. - Southwest Naturalist 26: 133-145.

[7] Darwin, C. (1859): El origen de las especies. Edition 1980. - EDAF, Madrid.

[8] Darwin, C. (1877): The various contrivances by which orchids are fertilised by insects. 2nd edition. - Appleton and Co., New York.

[9] Fleming, T., Holland, J. (1998): The evolution of obligate pollination mutualisms: Senita cactus and senita moth. - Oecologia 114: 368-375.

[10] Giurfa, M. (1991): La abeja recolectora de néctar. - Ciencia Hoy 2: 35-41.

[11] Gomez, J., Zamora, R. (1999): Generalization vs. specialization in the pollination system of Hormathophylla spinosa (Cruciferae). - Ecology 80: 796-805.

[12] Gomez, J., Zamora, R. (2000): Spatial variation in the selective scenarios of Hormathophylla spinosa (Cruciferae). - American Naturalist 155: 657-668.

[13] Greatti, M., Barbatini, R. (1997): Kiwi: uso de las abejas en la polinización. - Vida apícola 82: 27-31.

[14] Hassell, M., Jennings, J. (1975): The dynamics of competition and predation. - Edward Arnold, London.

[15] Hemborg, A., Despres, L. (1999): Oviposition by mut. seed-parasitic pollinators and its eff on annual fitness of single- and multi-flowered host plants. - Oecologia 120: 427-436.

[16] Herre, E., West, S. (1997): Conflict of interest in mutualism: documenting the elusive figwasp-seed tradeoff. - Proceed. of the Royal Society of London Ser. B 264: 1501-1507. 
[17] Herre, E., Knowlton, N., Mueller, U., Rehener, A. (1999): The evolution of mutualisms: exploring the paths between conflict and cooperation. - TREE 14: 49-53.

[18] Herrera, C., Jordano, P., Guitian, J., Traveset, A. (1998): Annual variability in seed production by woody plants and the masting concept: Reassessment of principles and relationship to pollination and seed dispersal. - American Naturalist 152: 576-594.

[19] Herrera, C. (2000a): Flower-to-seedling consequences of different pollination regimes in an insect-pollinated shrub. - Ecology 81: 15-29.

[20] Herrera, C. (2000b): Individual differences in progeny viability in Lavandula latifolia: a long-term field study. - Ecology 81: 2170-2176.

[21] Irwin, R., Brody, A. (1998): Nectar robbing in Ipomopsis aggregata: Effects on pollinator behavior and plant fitness. - Oecologia 116: 519-527.

[22] Kameyama, T., Harrison, R., Yamamura, N. (1999): Persistence of a fig wasp population and evolution of dioecy in figs: A simulation study. - Res. on Pop. Ecology 41: 243-252.

[23] Knuth, P. (1906): Handbook of flower pollination. - Oxford University Press, Oxford.

[24] Kostitzin, V. (1934): Symbiose, parasitisme et évolution. - Hermann, Paris.

[25] Lamborn, E., Ollerton, J. (2000): Experimental assessment of the functional morphology of inflorescences of Daucus carota (Apiaceae): Testing the 'fly catcher effect'. Functional Ecology 14: 445-454.

[26] López-Calleja, M., Fernández, M. (2001): ¿Usan los picaflores insectos sólo como fuente proteica? El caso de Sephanoides sephanoides. - In: I Reunión Binacional de Ecología Argentino-Chilena. ASAE, Bariloche.

[27] Margalef, R. (1995): Ecología. - Omega, Barcelona.

[28] May, R. (1981): Models for two interacting populations. - In: May R, (ed.) Theoretical Ecology. Pages 78-104. Sinauer Associates, Sunderland.

[29] Müller, H. (1883): The fertilization of flowers. - Macmillan, London,

[30] Muñoz Rogríguez, A. (1997): Girasol: polinización con ab. melíf. - Vida apíc. 84: 14-17.

[31] Pellmyr, O., Thompson, J., Brown, J., Harrison, R. (1996): Evolution of pollination and mutualism in the yucca moth lineage. - The American Naturalist 148: 827-847.

[32] Peters, R. (1983): The ecological implications of body size. - Cambridge University Press, Cambridge.

[33] Pleasants, J., Chaplin, S. (1983): Nectar production rates of Asclepias quadrafolia: causes and consequences of individual variations. - Oecologia 59: 232-238.

[34] Real, L., Rathcke, B. (1988): Patterns of individual variability in floral resources. Ecology 69: 728-735.

[35] Real, L., Marschall, E., Roche, B. (1992): Individual behavior and pollination ecology: Implications for the spread of sexually transmitted plant diseases. - In: DeAngelis, D.L., Gross, L.J., (eds.) Individual-based models and approaches in ecology. Populations, communities and ecosystems. Pages 492-508. Chapman \& Hall, New York - London.

[36] Robertson, A,. Kelly, D., Ladley, J., Sparrow, A. (1999): Effects of pollinator loss on endemic New Zealand mistletoes (Loranthaceae). - Conservation Biology 13: 499-508.

[37] Traveset, A. (1999): The importance of mutualisms for biodiversity conservation in insular ecosystems. - Revista Chilena de Historia Natural 72: 527-538.

[38] van Baalen, M., Jansen, V. (2001): Dangerous liasons: the ecology of private interest and common good. - Oikos 95: 211-224.

[39] Waser, N., Prise, M. (1983a): Pollinator behavior and natural selection for flower color in Delphinium nelsonii. - Nature 302: 422-424.

[40] Waser, N., Prise, M. (1983b): Optimal and actual outcrossing in plants, the nature of plant-pollinator interaction. - In: Jones, C.E., Little, R.J., (eds.) Handbook of Pollination Biology. Pages 341-359. Van Nostrand-Reinhold, New York.

[41] Waser, N. (1993): The adaptative nature of floral traits: ideas and evidence. - In: Real, L.A., (ed.) Pollination Biology. Pages 241-285. Academic Press, New York. 